KNOWLEDGE DISCOVERY IN THE SOCIAL SCIENCES 



\title{
KNOWLEDGE DISCOVERY IN THE SOCIAL SCIENCES
}

\author{
A Data Mining Approach
}

Xiaoling Shu

Ф UNIVERSITY OF CALIFORNIA PRESS 
University of California Press

Oakland, California

(C) 2020 by Xiaoling Shu

Library of Congress Cataloging-in-Publication Data

Names: Shu, Xiaoling, 1968- author.

Title: Knowledge discovery in the social sciences : a data mining approach / Xiaoling Shu.

Description: Oakland, California : University of California Press, [2020] | Includes bibliographical references and index.

Identifiers: LCCN 2019024334 (print) | LCCN 2019024335 (ebook) | ISBN 9780520339996 (cloth) |

ISBN 9780520292307 (paperback) | ISBN 9780520965874 (ebook)

Subjects: LCSH: Social sciences—Research—Data processing. | Data mining.

Classification: LCC H61.3 .S49 2020 (print) | LCC H61.3 (ebook) | DDC 300.285/6312—dc23

LC record available at https://lccn.loc.gov/2019024334

LC ebook record available at https://lccn.loc.gov/2019024335

Manufactured in the United States of America

$\begin{array}{llllllllll}29 & 28 & 27 & 26 & 25 & 24 & 23 & 22 & 21 & 20\end{array}$

$\begin{array}{llllllllll}10 & 9 & 8 & 7 & 6 & 5 & 4 & 3 & 2 & 1\end{array}$ 
To Casey, Kina, and Dong with love and gratitude 
